\title{
On Wannier exciton 2D localization in hydrogen intercalated InSe and GaSe layered semiconductor crystals
}

\author{
Yu.I. Zhirko' ${ }^{1}$ I.P. Zharkov' ${ }^{1}$ Z.D. Kovalyuk ${ }^{2}$, M.M. Pyrlja ${ }^{2}$, V.B. Boledzyuk ${ }^{2}$ \\ 1 Institute of Physics, NAS of Ukraine, 46, prospect Nauky, 03037 Kyiv, Ukraine \\ E-mail: zhirko@nas.gov.ua \\ 2 Chernivtsy Branch of Frantsevych Institute for Problems of Material Science, NAS of Ukraine, 5, Irina Vilde str., \\ 58001 Chernivtsy, Ukraine
}

\begin{abstract}
Exciton absorption spectra of layered InSe and $\mathrm{GaSe}$ crystals electrochemically intercalated by hydrogen were investigated. It was found that the observed at $T=80 \mathrm{~K}$ non-monotonic shift of the exciton absorption peak $n=1$ with growing hydrogen concentration $x$ (here $x$ is the amount of hydrogen atoms per a formula unit of a crystal matrix) stems from the increasing dielectric permittivity of the crystal $\varepsilon_{0}$ due to availability of hydrogen in the van der Waals gap. Growth of $\varepsilon_{0}(x)$ results in decrease of the exciton binding energy but, at larger $x$ concentrations (when the anisotropy parameter $\varepsilon^{*}(x)$ grows), 2D localization of exciton motion in the crystal layer plane takes place, which causes reduction and then, at $x>1$, stabilization of sizes both for the exciton and quantum well.
\end{abstract}

Keywords: exciton absorption, layered crystal, InSe, GaSe, quantum well.

Manuscript received 30.09.04; accepted for publication 16.12.04.

\section{Introduction}

$\mathrm{A}^{3} \mathrm{~B}^{6}$ semiconductor compounds including layered $\gamma$ InSe and $\varepsilon$-GaSe crystals attract investigator's interest due to the fact that heterostructures of these crystals possess a good photosensitivity and find their application in solar cells [1-4]. By convention, the great attention of researchers is paid to intercalation, that is to insertion of atoms and molecules into the interlayer space of a layered crystal (the so-called van der Waals gap) where weak molecular bonds act between crystal layers. Appearance of great impurity amounts in the gap can essentially change the intrinsic electrical resistance of the crystal from semiconductor to metallic values in the direction perpendicular to the layer, can cause a growth of the crystal dielectric permeability [5], appearance of supplementary (local) interlayer vibrations in its phonon spectrum [6] and even crystal exfoliation when intercalation reaches a sufficient level [7].

Layered $\gamma$-InSe and $\varepsilon$-GaSe crystals are rather promising for hydrogen energetics as operating elements in systems for hydrogen accumulation [8-10]. The concentration of hydrogen in them can reach values $x=$ $3 . .5$, where $x$ is the amount of introduced atoms per one formula unit of the crystal bulk.

In this work, we report on the investigations of the hydrogen intercalation influence on optical properties of
$\gamma$-InSe and $\varepsilon$-GaSe crystals in the region of $n=1$ exciton resonance.

\section{Experimental part}

Layered InSe and GaSe crystals belong to $\mathrm{A}^{3} \mathrm{~B}^{6}$ binary compounds. The layers in them consist of four Se$\operatorname{In}(\mathrm{Ga})-\operatorname{In}(\mathrm{Ga})-\mathrm{Se}$ sheets with three Se atoms coordinated to one $\operatorname{In}(\mathrm{Ga})$ atom that corresponds to spatial group $D_{3 h}^{1}$ (see Fig. 1). The covalent $\operatorname{In}(\mathrm{Ga})-\operatorname{In}(\mathrm{Ga})$ bonds are oriented perpendicular to the layers. Bridgman grown InSe and GaSe crystals have four ( $\beta-, \delta-, \varepsilon^{-}$, and $\left.\gamma-\right)$ polytypes $[11,12]$, which differ by sequence of layers stacking. Thus, bulk InSe single crystals are related to the $\gamma$-polytype with the rhombohedral (trigonal) crystal structure (space group $C_{3 v}^{5}$, one $\mathrm{In}_{2} \mathrm{Se}_{2}$ molecule in the primitive unit cell). The conventional hexagonal unit cell extends over three layers and consists of three $\operatorname{In}_{2} \mathrm{Se}_{2}$ molecules. GaSe single crystal has the $\varepsilon$-polytype structure. It belongs to the hexagonal crystal structure (space group $D_{3 h}^{1}$ ) primitive unit cell of which consists of two $\mathrm{Ga}_{2} \mathrm{Se}_{2}$ molecules (eight atoms) located within two crystal layers [12].

Lattice parameters of $\gamma$-InSe and $\varepsilon$-GaSe crystals are well known. For $\varepsilon$-GaSe they are: $C_{0}=15.95 \AA$ and $a_{0}=3.755 \AA$ [13]. For $\gamma$-InSe they are: $C_{0}=25.32 \AA$ and 

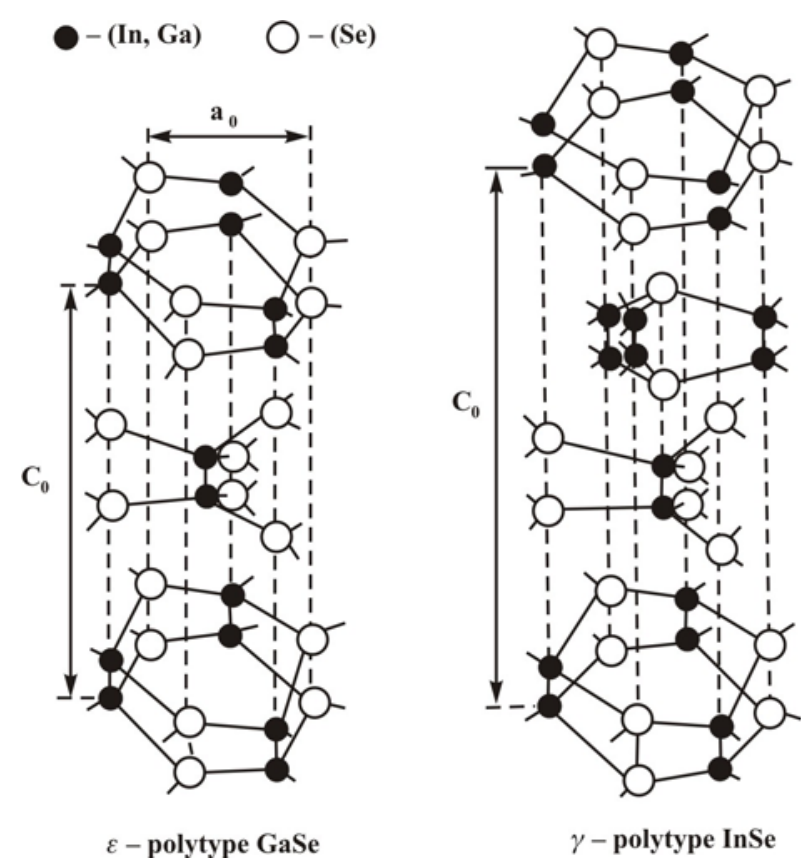

Fig. 1. The crystal structure and stacking of a crystal layers in $\gamma$-InSe and $\varepsilon$-GaSe.

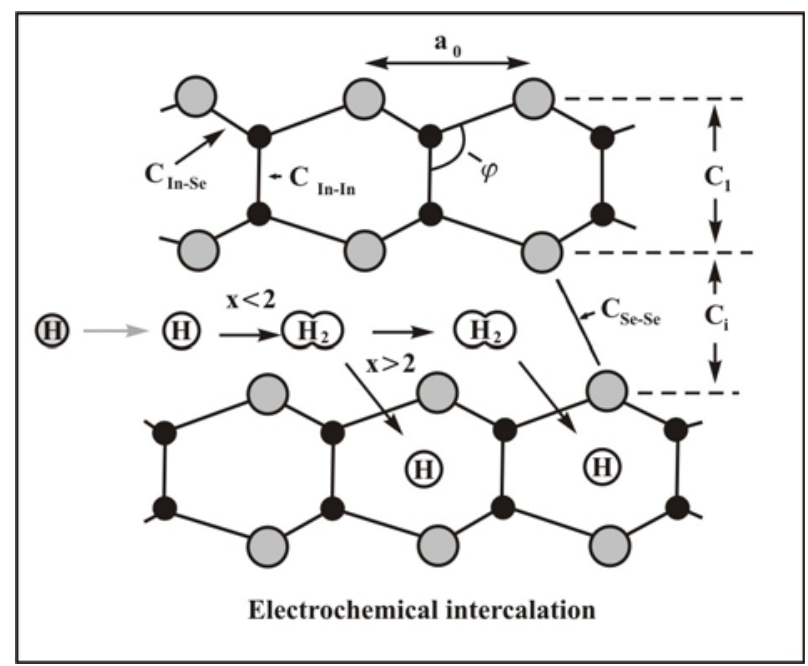

Fig. 2. Scheme demonstrates processes of hydrogen intercalation in layered crystals.

$a_{0}=4.001 \AA$. Interlayer nearest-neighbour distances in layers (see Fig. 2) are $C_{\mathrm{In}-\mathrm{In}}=2.79 \AA$ and $C_{\mathrm{In}-\mathrm{Se}}=2.65 \AA$, respectively; the angle $\varphi=119.3^{\circ}$, the layer thickness $C_{1}=5.36 \AA$, the distance between layers $C_{i}=3.08 \AA$ and their interlayer distance $C_{\mathrm{Se}-\mathrm{Se}}=3.80 \AA[14,15]$.

\subsection{Intercalation}

Hydrogen intercalation of single crystal $\gamma$-InSe and $\varepsilon$-GaSe samples of 10 to $20 \mu \mathrm{m}$ thickness was carried out (like as in [10]) using the electrochemical method from 0.1 normal solution of hydrochloric acid with sweeping electric field in the galvanostatic regime. A special choice of the optimal current density enabled to obtain homogeneous in their composition intercalated with hydrogen $\mathrm{H}_{x} \mathrm{InSe}$ and $\mathrm{H}_{x} \mathrm{GaSe}$ samples within the range of concentrations $0<x \leq 2$. The hydrogen concentration in $\mathrm{H}_{x} \mathrm{InSe}$ was determined via the quantity of electrical charge transported through the sample placed into the special cell. We assume that atomic hydrogen enters into the van der Waals gap where $\mathrm{H}_{2}$ molecules creates that occupy positions schematically shown in Fig. 2.

In the present work, there was carried out the X-ray diffraction study of $\mathrm{H}_{x} \mathrm{GaSe}$ crystals at $300 \mathrm{~K}$. It was shown that the parameter $C_{0}$ grows with $x$ and increases by $0.031 \AA$ at $x=1.0$. The same data about the growing $C_{0}$ parameter were observed also in [5] when GaSe crystals were intercalated with fluorine.

$\mathrm{X}$-ray diffraction data are in a good agreement with our calculations for InSe, which, according to Clapeyron's equation $P=N k_{\mathrm{B}} T$ for the ideal gas pressure, shown that, at $x=2$, in the interlayer space (equal to $9.4 \mathrm{MPa}$ at $T=80 \mathrm{~K}$ and $35.4 \mathrm{MPa}$ at $T=300 \mathrm{~K}$ ) and in accordance to [15] pressure of $\mathrm{H}_{2}$ caused an increase of the interlayer distance and the parameter $C_{0}$ growth by $0.024 \pm 0.001 \AA$ at $T=300 \mathrm{~K}$ and by $0.009 \pm 0.001 \AA$ at $T=80 \mathrm{~K}$. Moreover, when $x=2$ and $T=80 \mathrm{~K}$ the rotation of $\mathrm{H}_{2}$ molecules in translationally ordered cites of the gap is practically absent (for $\mathrm{H}_{2} T_{r}=h^{2} / 8 \pi^{2} J k_{\mathrm{B}}=90 \mathrm{~K}$, where $J$ is the rotational moment of inertia) and this state may be treated as the state of a "quasi-liquid monolayer".

It was also shown that geometrical sizes of $\mathrm{H}_{2}$ molecules and van der Waals gap have such values that causes the quantum-size effect. This effect together with a Pauli principle (for Fermi particles such as molecular para- and ortohydrogen in discrete states) caused a strong repulsion of more than one $\mathrm{H}_{2}$ molecule in translationally ordered cites, shown in Fig. 2. This repulsion leads to the condition that, at $x>2$, the only one molecule of $\mathrm{H}_{2}$ remains in this van der Waals gap cites, while abundant atomic hydrogen should penetrate into interstices of crystal layers.

\subsection{Absorption}

Investigations of $\mathrm{H}_{\mathrm{x}} \mathrm{InSe}$ and $\mathrm{H}_{x}$ GaSe crystals absorption spectra were carried out at $T=80 \mathrm{~K}$ using the $1 / 2 \mathrm{~m}$ spectrometer with 600 grooves $/ \mathrm{mm}$ diffraction grating and resolution not worse than $0.5 \mathrm{meV}$. As a light source, we used the incandesent filament lamp supplied from the stabilized direct current unit.

InSe and GaSe crystals are characterized with a weak interaction of 3D Wannier excitons with homopolar optical A'-phonons $[16,17]$. Therefore, when calculating the exciton absorption spectra (see Fig. 3), we took into consideration effects of broadening the exciton states using the standard convolution procedure (see in [16]) for theoretical values of $\alpha(\hbar \omega)$ the absorption coefficient in the Elliott model [18] with $f(\hbar \omega)=\Gamma /\left[\pi\left(E^{2}+\Gamma^{2}\right)\right]$ the Lorentzian function in the 

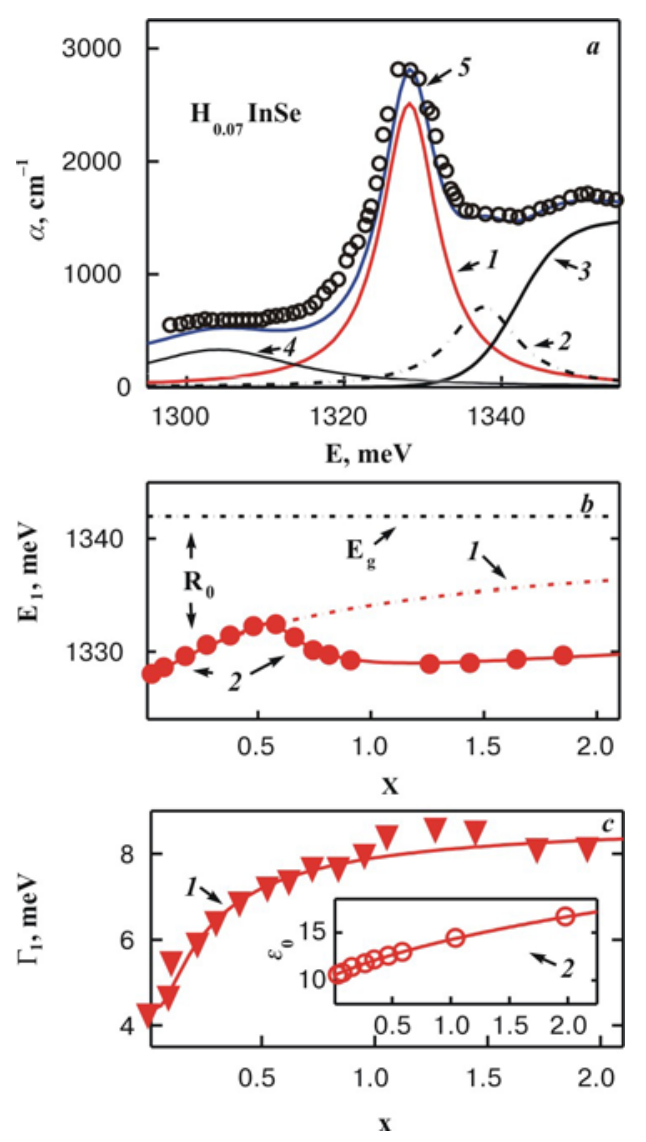

Fig. 3. Exciton absorption spectra of $\mathrm{H}_{x} \mathrm{InSe}$ at $T=80 \mathrm{~K}$.

a - experimental (open circles) and calculated (curve 5) exciton absorption spectra of $\mathrm{H}_{0.07} \mathrm{InSe}$ crystal at $T=80 \mathrm{~K}$. Curves $1-4$ are the bands of $n=1,2$ exciton states, band-to-band transition and shallow donor states;

b - circles show experimental shift of $n=1$ exciton absorption band maximum $E_{1}$ on $x$ for $\mathrm{H}_{x} \mathrm{InSe}$. The line denoted as $E_{g}$ shows the stable energy position of $E_{\mathrm{g}}$ on $x$. Curve 1 is plotted in accordance with the assumption of increase of the dielectric permeability $\varepsilon_{0}$. Curve $2-$ with account of exciton localization in the layer plane. $R_{0}$ show a decrease of the exciton binding energy with $x$ when exciton localization is ignored;

$\mathrm{c}-$ solid triangles show the experimental $\Gamma_{1}$ dependence on $x$. Curve 1 is plotted in accordance with Eq. (1) . In insert, open circles show the experimental $\varepsilon_{0}$ dependence on $x$. Curve 2 is plotted in accord to Eq. (5).

Toyozawa model [19], where $\Gamma$ is the half-width at halfmaximum which is usually associated with the lifetime $\hbar / 2 \Gamma$.

Basing on the performed calculations, it was shown that polaritonic effects associated with the growth of the integral intensity of $n=1$ exciton absorption band $K$ does not take place in our crystals at $T=80 \mathrm{~K}$. Therefore, we suppose that $K \cong K^{0}$, where $K^{0}$ is the classical value. Thus, in Fig. 3, it was shown the experimental (open circles) and calculated (curve 5) exciton absorption spectra of $\mathrm{H}_{0.07} \mathrm{InSe}$ crystal at $T=80 \mathrm{~K}$.

At the same time, when introducing hydrogen into the interlayer space, one can observe the following peculiarities:

1. In Fig. 3b, depicted by circles is the experimental dependence of the maximum position for $n=1$ exciton absorption bands $E_{1}$ on $x$ for $\mathrm{H}_{x} \mathrm{InSe}$ crystals. Within the range $0<x \leq 0.5$, the peak energy position shifts upward by $\Delta E_{1}=4.5 \mathrm{meV}$ from $E_{1}=1327.5 \mathrm{meV}$ up to $E_{1}=1332.0 \mathrm{meV}$; at $0.5<x \leq 1$, it goes downward to the energy $E_{1}=1329.5 \mathrm{meV}$; the further increase of hydrogen amount in the interlayer space does not result in changing $E_{1}(x)$ values.

2. In Fig. 3c, the curve of solid triangles means the change of the halfwidth $\Gamma_{1}$ of the $n=1$ excitonic absorption band on $x$ for $\mathrm{H}_{x} \mathrm{InSe}$ crystals. It can be seen that $\Gamma_{1}$ increases with $x$ within the range $0<x \leq 1$ and becomes practically constant at $x>1$. The experimental $\Gamma_{1}(x)$ dependence can be expressed by the following function:

$$
\Gamma_{1}(x)[\mathrm{meV}]=\Gamma_{1}\left\{1+1 / \exp \left[1 /\left(\Gamma_{1} x\right)\right]\right\},
$$

where $\Gamma_{1}=4.4 \mathrm{meV}$.

The marked peculiarities of the nonlinear shift inherent to the $n=1$ exciton absorption band maximum at $T=80 \mathrm{~K}$ with hydrogen concentration are also observed in $\mathrm{H}_{x} \mathrm{GaSe}$ crystals. However, in $\mathrm{H}_{x} \mathrm{GaSe}$ these phenomena are less pronounced. Thus, within the range $0<x \leq 0.8$, the peak energy position shifts upward by $\Delta E_{1}=3.0 \mathrm{meV}$ from $E_{1}=2099.0 \mathrm{meV}$ up to $E_{1}=2102.0 \mathrm{meV}$; at $0.8<x \leq 1.5$, one can observe its shift down to the energy $E_{1}=2100.5 \mathrm{meV}$; the further increase of hydrogen amount in the interlayer space does not result to changing $E_{1}(x)$ values. Simultaneously with $E_{1}(x)$ nonlinear shift experimental dependence $\Gamma_{1}$ also increases with $x$ within the range $0<x \leq 1.5$ and becomes practically constant at $x>1.5$. For $\mathrm{H}_{x} \mathrm{GaSe}$, the experimental dependence $\Gamma_{1}(x)$ is in accordance with Eq. (1) for $\Gamma_{1}=3.6 \mathrm{meV}$.

\section{Discussion}

At first, we shall carry out a discussion taking into account the experimental data obtained for InSe crystals and then generalize them to InSe and GaSe crystals.

The observed in InSe crystals shortwave shift of $E_{1}$ with $x$ growth could be explained in a most simple way by a change of the forbidden gap $E_{g}$ with the pressure growth, this pressure being caused by hydrogen in the interlayer space. Moreover, as shown in $[14,20]$, the $E_{g}(P)$ dependence in $\gamma$-InSe crystals is non-monotonic. For example, at the hydrostatic pressures of $10^{5}$ up to 
$4.2 \cdot 10^{8} \mathrm{~Pa}$ in $\gamma$-InSe crystals, $E_{g}$ decreases by $3.3 \mathrm{meV}$, but with the following pressure increase it grows, too.

As shown in the previous part, the hydrogen concentration increase up to $x=2$ gives rise to the pressure between layers up to $P=9.4 \mathrm{MPa}$ at $T=80 \mathrm{~K}$, which stimulates an increase of the van der Waals gap. Making extrapolation in accord with the analytical dependences $[14,20]$, we obtained $E_{g}$ growth by $0.2 \mathrm{meV}$ at $P=9.4 \mathrm{MPa}$. At $x=0.5$ and $T=80 \mathrm{~K}$, this increase is $\Delta E_{g}=0.05 \mathrm{meV}$. The shift $\Delta E_{g}=4.5 \mathrm{meV}$ could take place at the pressure of molecular hydrogen on layer packages $P=205 \mathrm{MPa}$. Indeed, hydrogen can provide this pressure at $x=12$ and $T=300 \mathrm{~K}$ but under the only condition that, for $x>2$, it does not penetrate into interstices of InSe layer packages and contains six $\mathrm{H}_{2}$ molecules in the cavity. Hence, we made the conclusion that the observed shortwave shift of $E_{1}$ by 4.5 meV cannot be caused by a baric shift of $E_{g}$.

It is also known [21] that an increase of the hydrostatic pressure in $\mathrm{A}^{3} \mathrm{~B}^{6}$ crystals, contrary to $\mathrm{A}^{2} \mathrm{~B}^{6}$ and $\mathrm{A}^{3} \mathrm{~B}^{5}$ ones, results in increasing $\varepsilon_{0} \|$ component of the crystal dielectric permeability. As it was shown by direct measurements of the capacitance [21], $\varepsilon_{0}{ }^{\|}$of InSe grows almost linearly by $15 \%$ within the range $10^{5}$ to $10^{9} \mathrm{~Pa}$. Thereof, in accord with the mean $\varepsilon_{0}$ value that is defined as $\varepsilon_{0}=\left[\varepsilon_{0}{ }_{0} \varepsilon_{0}{ }^{\perp 2}\right]^{1 / 3}$ in anisotropic layered crystals (when, accordingly to [16], $\varepsilon_{0}{ }^{\perp}=10.9, \varepsilon_{0}{ }^{\|}=9.9$, and indexes $\|$, $\perp$ correspond to parallel and perpendicular orientations relatively to the optical axis $C$ ), $\varepsilon_{0}$ grows by $5 \%$. This $\varepsilon_{0}$ increase should result in decreasing the exciton binding energy $R_{0}$. Indeed, the decrease of $R_{0}$ from $14.2 \mathrm{meV}$ down to $12.9 \pm 0.4 \mathrm{meV}$ at $P=1 \mathrm{GPa}$ was reported in [14]. The $R_{0}$ value obtained in [14] and $\varepsilon_{0}$ observed in [21] increase by $5 \%$ which are in a good agreement with the well-known dependence

$R_{0}[\mathrm{meV}]=13605 \mu / \varepsilon_{0}^{2}$

combining $R_{0}$ with $\varepsilon_{0}$ and $\mu$ - reduced exciton mass (in InSe $\mu=\left[m_{e}^{-1}+m_{h}^{-1}\right]^{-1}=0.12 m_{0}$ [16], where $m_{0}$ is the mass of the free electron).

Extrapolating data $[14,21]$ onto our case of the van der Waals gap growth, one can say that, at $x=2$ $(P=9.4 \mathrm{MPa}), \varepsilon_{0}$ should decrease by $0.7 \%$ and $R_{0}$ increase by $0.2 \mathrm{meV}$, which at $x=0.5$ and $\Delta \varepsilon_{0}(P)=0.2 \%$ results in $\Delta R_{0}(P)=0.06 \mathrm{meV}$ and the respective longwave shift of $E_{1}$.

The performed analysis of obtained by various authors data upon the pressure influence on the energy structure of InSe crystal enables one to draw the conclusion that the shortwave shift of $E_{1}$ by $4.5 \mathrm{meV}$ cannot be reasonably explained by $R_{0}(P), \varepsilon_{0}(P)$ and $E_{g}(P)$ dependences, as $R_{0}(P)$ and $E_{g}(P)$ contributions into this shift are opposite at $x=0.5(P=2.4 \mathrm{MPa})$ and their total contribution is only $0.01 \mathrm{meV}$, which is considerably lower than the observed one.
At the same time, this behaviour of exciton parameters $E_{1}(x)$ and $\Gamma_{1}(x)$ gives grounds to deem that the shortwave shift of the exciton absorption band is caused by the $\varepsilon_{0}$ increase stemming from the hydrogen presence in the interlayer space, which results in $R_{0}$ decrease in accord with Eq. (2).

To make our discussion convenient, we shall describe the $\varepsilon_{0}$ increase caused by molecular hydrogen in the interlayer space introducing an additive to $\varepsilon_{0}^{\|_{0}}$ parameter $\varepsilon^{*}(x)$ increasing with $x$ and characterizing the degree of dielectric permeability anisotropy for the medium comprised by the exciton in the following form:

$\varepsilon_{0}(x)=\left[\varepsilon_{0}{ }^{\perp 2} \varepsilon_{0}{ }^{\|} \varepsilon^{*}(x)\right]^{1 / 3} \equiv \varepsilon_{0}\left[\varepsilon^{*}(x)\right]^{1 / 3}$,

where parameter

$\varepsilon^{*}(x)=\prod_{j=1}^{w} \varepsilon_{i}(x) \equiv \varepsilon_{i}(x)^{w}$.

$w$ is the degree index characterizing the quantity of interlayer space comprised by the exciton diameter $d_{\mathrm{exc}}$, and $\varepsilon_{i}(x)$ is the dependent on $x$ dielectric permeability of the van der Waals gap, which is equal to that of vacuum $\varepsilon_{i}(0)=\varepsilon_{v}=1$ in the case of pure InSe.

To simplify our estimation procedure, we shall consider that hydrogen introduction into the interlayer space changes neither $E_{g}$ nor $\mu$. Then, the shortwave shift of $E_{1}(x)$ in the range $0<x \leq 0.5$ (see Fig. $3 b$ ) is caused by $R_{0}$ decrease from 14.5 down to $10.0 \mathrm{meV}$, which results in practically linear growth of the obtained experimental $\varepsilon_{0}(x)$ values from 10.5 to 12.6 in accord with Eq. (2) (see insert of Fig. 3c) and is described rather well by the following dependence:

$\varepsilon_{0}(x)=10.5(1+1.5 x)^{1 / 3}$

shown by the curve 2 . The curve 1 in Fig. $3 b$ represents the dependence $R_{0}(x)$ for $0<x \leq 2$ obtained according to Eq. (2) in the case when $\varepsilon_{0}(x)$ corresponds to Eq. (5). As it can be seen, the curve 1 well describes the experimental dependences $R_{0}(x)$ within the range $0<x \leq 0.5$. In accord with Eq. (3), $\varepsilon_{0}{ }_{0}(x)$ increases from 9.9 up to 16.9 due to the growth of parameter $\varepsilon^{*}(x)$ from $\varepsilon_{v}=1$ up to 1.7 . In accordance with the expression

$a_{\mathrm{exc}}[\mathrm{nm}]=0.053 \varepsilon_{0}(x) / \mu$,

where $a_{\text {exc }}$ is the exciton radius, the exciton diameter grows linearly with changing $\varepsilon_{0}(x)$ from 9.51 up to $11.43 \mathrm{~nm}$, which is equivalent to the increase of the exciton diameter from $d_{\text {exc }}=11.3$ up to 13.5 crystal layers (layer thickness of InSe $d_{\text {layer }}=8.44 \AA$ ). Thereof, for $w=13$ in accord to Eq. (4) at $x=0.5(P=2.3 \mathrm{MPa})$ and $T=80 \mathrm{~K}$, one can deduce $\varepsilon_{i}=1.04$. 


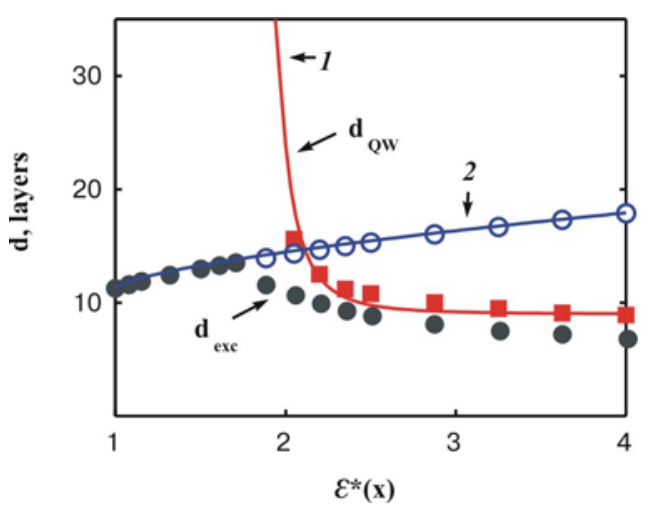

Fig. 4. Solid squares are the experimental values for the quantum well thickness $d_{\mathrm{QW}}$ on the $\varepsilon^{*}(x)$ parameter for $\mathrm{H}_{x} \mathrm{InSe}$ at $T=80 \mathrm{~K}$. Circles and solid circles are experimental dependences for the exciton diameter $d_{\mathrm{exc}}$ on the $\varepsilon^{*}(x)$ parameter without and with the account of exciton localization. Curve 1 and 2 are plotted in accord to Eqs (10) and (6).

Considering Eq. (5) defining the dependence of $\varepsilon_{0}$ on $x$, which is associated with hydrogen introduction into interlayer space, we may perform extrapolation of $\varepsilon_{0}(x)$ to $x=1$. Obtained data will be as follows: $\varepsilon_{0}=14.25$ and parameter $\varepsilon^{*}=2.5 . R_{0}$ should decrease down to $6.45 \mathrm{meV}$, while the exciton diameter should increase to $d_{\mathrm{exc}}=12.9 \mathrm{~nm}\left(d_{\mathrm{exc}}=15.3\right.$ crystal layers $)$. For $w=15$ at $x=1(P=4.7 \mathrm{MPa})$ and $T=80 \mathrm{~K}$, one can determine $\varepsilon_{i}=1.062$.

In the similar way, for $x=2$ we can obtain $\varepsilon_{0}=16.67$ and $\varepsilon^{*}=4.0, R_{0}=5.73 \mathrm{meV}, d_{\mathrm{exc}}=15.1 \mathrm{~nm}$ $\left(d_{\mathrm{exc}}=17.9\right.$ crystal layers $)$. For $w=18$ at $x=2$ $(P=9.4 \mathrm{MPa})$ and $T=80 \mathrm{~K}$, one can determine $\varepsilon_{i}=1.081$. It is evident that $\varepsilon_{i}$ value also grows with $x$ and begins to approach to experimental data inherent to the dielectric permeability of condensed hydrogen [22], which is 1.253 and 1.230 at the temperatures 14 and $20.5 \mathrm{~K}$, respectively.

The obtained numeric values for dependences of parameter $\varepsilon^{*}$ and $\varepsilon_{i}$ on $x$ are well described by the following analytical expressions:

$\varepsilon^{*}(x)=\varepsilon_{v}+1.5 x$

and

$\varepsilon_{i}(x)=\varepsilon_{v}+0.06 x^{1 / 2}$,

where $\varepsilon_{v}=1$. It is seen that $\varepsilon_{i}$ growth as a whole causes increasing the crystal dielectric permeability that leads to the experimentally observed $R_{0}$ decrease in complete agreement with Eqs (2) and (5), as shown by the curve 1 in Fig. 3b.

However, at $0.5<x<1, E_{1}(x)$ shift changes its sign by the opposite one, and at $1<x<2 E_{1}$ position is stabilized. This behaviour of $E_{1}(x)$ allows us to posit that $\varepsilon_{i}(x)$ increase causes a growth of $a_{\mathrm{exc}}$. As a consequence, parameter $\varepsilon^{*}(x)$ characterizing the degree of anisotropy inherent to medium comprised by the exciton grows so much that at $\varepsilon^{*}=1.7(x=0.5)$ the exciton motion is localized in the plane of layers. Further increase of $\varepsilon_{i}(x)$ causes reducing the exciton radius and quantum well (QW) width $d_{\mathrm{QW}}$.

In Fig. 4, squares shown the dependence of the localized exciton QW width on the parameter $\varepsilon^{*}(\boldsymbol{x})$ obtained in accordance with

$E=\frac{\pi^{2} \hbar^{2}}{2 d^{2} M}$

and experimental $\Delta E_{1}(x)$ data. For convenience, we chose the thickness of the crystal layer as a unit of the $d_{\mathrm{QW}}$. Open circles show the dependence of the exciton diameter $d_{\mathrm{exc}}$ on parameter $\varepsilon^{*}(x)$ without taking into account exciton localization, while solid circles do that considering the localization in the QW.

As seen from this figure, within the range of parameter values $1<\varepsilon^{*}(x)<1.7$ the QW does not appear $\left(d_{\mathrm{QW}}=\infty\right)$, and we deal with the ordinary growth of $d_{\mathrm{exc}}$ (open circles) bound by $\varepsilon_{i}(x)$ increase. At $1.7<\varepsilon^{*}(x)<2.5$, strong localization of the exciton takes place in the layer plane, and the QW narrows from 107 down to 12.8 layers. At the same time, $d_{\mathrm{exc}}$ decreases from 13.5 down to 8.8 layers.

Further $x$ increase, in accord with Eq. (7), causes $\varepsilon^{*}(x)$ parameter growth from 2.5 up to 4.0 and decrease the QW and exciton diameter down to $d_{\mathrm{QW}}=12.8$ layers and $d_{\text {exc }}=10.55$ layers. $R_{0}$ and $d_{\text {exc }}$ are stabilized at the final level, and the exciton is localized in the crystal layer plane. Experimental data of $d_{\mathrm{QW}}\left(\varepsilon^{*}\right)$, where [see Eq. (7)] $\varepsilon^{*}=f(x)$, is well approximated by the following expression:

$d_{\mathrm{QW}}\left(\varepsilon^{*}\right)[$ layers $]=9+0.4 /\left(\varepsilon^{*}(x)-1.7\right)^{3}$

represented by the curve 1 in Fig. 4 .

Note that, at $\varepsilon^{*}(x)>1.7$, numeric $d_{\mathrm{exc}}$ values were obtained in approximation that increasing the parameter up to $\varepsilon^{*}(x)>1.7$ should be followed by the condition $\varepsilon_{i}(x)^{w} \leq 1.7$. As $\varepsilon_{i}(x)$ value grows with $x$, the quantity of layers comprised by the exciton $w$ should be decreased.

This statement is confirmed by the experimental dependence of the $n=1$ exciton absorption band halfwidth $\Gamma_{1}$ on $x$ depicted in Fig. 3c. Indeed, the experimentally observed $d_{\text {exc }}$ growth in the range $x<0.5$ results in increasing the probability of exciton scattering by point defects and increasing the initial value of homogeneous broadening the ground as well as excited exciton states $\Gamma_{i}^{\prime}(0)=g^{2}\left[\hbar \Omega\left(R_{0} / i^{2}-\hbar \Omega\right)\right]^{1 / 2}$. Despite the more smooth $\Gamma_{i}^{2}(T)$ dependence, the total exciton scattering rises at the low temperature $(T=80 \mathrm{~K})$, which results in experimentally observed $\Gamma_{1}$ growth. In the range $x>0.5$, the exciton localization causes $\varepsilon_{i}(x)$ growth and $d_{\text {exc }}$ decrease, which results in stabilization of exciton and QW sizes and reaching a stationary value of the probability for excitons to be scattered by defects. 
The experimental $\Gamma_{1}(x)$ dependence is in accordance with Eq. (1) depicted by the curve 1 . Here $\Gamma_{1}=4.4 \mathrm{meV}$.

Comparing InSe with GaSe crystals shows that, in GaSe crystals, described above phenomena of $E_{1}$ nonlinear behaviour with $x$ are less expressed and more delayed at the large hydrogen concentration. The main reason of these differences is that, in GaSe crystal, exciton diameter ( $d_{\mathrm{exc}}=9$ crystal layers) is less than that in InSe crystal, where exciton diameter $d_{\text {exc }}$ envelop 11.3 crystal layers. It is clear that, at a smaller exciton diameter, the described phenomena require larger hydrogen concentrations. That is why, in $\mathrm{H}_{x} \mathrm{GaSe}$ crystals, the effects of reduction of the exciton binding energy and subsequent exciton localization with an increase of hydrogen in van der Waals gap are more delayed and much less expressed.

Oneself paid attention that, for $T=80 \mathrm{~K}$ and $x=0$, the experimental halfwidth of the $n=1$ exciton absorption band $\Gamma_{1}$ in InSe crystals is greater than that in GaSe crystals. First of all, it is connected with the fact that, in GaSe crystals, the energy of homopolar phonon is essentially higher than that in InSe crystals and hence its contribution to $\Gamma_{1}$ broadening as well as to growth of the integral intensity of $n=1$ exciton absorption band up to classical values is manifested at higher temperatures.

\section{Conclusions}

The performed investigations of hydrogen intercalation in layered InSe and GaSe crystals have shown that hydrogen in the atomic state enters into the van der Waals gap and forms $\mathrm{H}_{2}$ molecules that, with the growth of hydrogen concentrations up to $x=2$, has a tendency to occupy translation ordered states in the gap and causes there a pressure resulting in experimentally observed and predicted by our calculations growth of the interlayer lattice parameter $C_{0}$.

At $x=2$ and $T<80 \mathrm{~K}$, this state of $\mathrm{H}_{2}$ in the gap can be treated as a "quasi-liquid monolayer". At $x>2$, atomic hydrogen begins to incorporate into interstices of the crystal lattice due to quantum-size effects arising in the gap and strong repulsion between $\mathrm{H}_{2}$ molecules.

It was found that the observed at $T=80 \mathrm{~K}$ nonmonotonic shift of the $n=1$ exciton absorption band peak with $x$ stems from the increasing dielectric permeability $\varepsilon_{0}$ of the crystal due to presence of $\mathrm{H}_{2}$ molecules in the gap. A growth of the exciton anisotropy parameter $\varepsilon^{*}(x)$ results in decrease of the exciton binding energy $R_{0}$. When $\varepsilon^{*}(x)$ exceeds the critical value $\varepsilon^{*} \leq 2,2 \mathrm{D}$ localization of exciton motion in the crystal layer plane followed by the growth of $R_{0}$ takes place, which causes reduction and then stabilization of sizes both for the exciton and quantum well. Due to the difference in exciton diameters $\left(d_{\text {exc }}^{\mathrm{GaSe}}<d_{\mathrm{exc}}^{\mathrm{InSe}}\right)$, this phenomena in GaSe crystals are more delayed and much less pronounced.

\section{Acknowledgements}

This work was partially supported by the Basic Research Fund of Ukraine (Project No F7/310-2001).

\section{References}

1. A.A. Lebedev, V.Yu. Rud', Yu.V. Rud', Photosensitivity of geterostructures porous siliconlayered $\mathrm{A}^{\mathrm{III}} \mathrm{B}^{\mathrm{VI}}$ semiconductors // Fiz. Tekhn. Polupr. 32 (3), p. 353-355 (1998).

2. V.N. Katerinchuk, Z.D. Kovalyuk, V.M. Kaminskii, Geterotransitions $p$-GaSe- $n$-recrystallized InSe // Pis'ma Zhurn.Tekhn. Fiz. 26 (2), p. 19-22 (2000).

3. J. Martines-Pastor, A. Segura, J.L. Valdes, Electrical and photovoltaic properties of indium-tinoxide/p-InSe/Au solar cells // J. Appl. Phys. 62 (4), p. 1477-1483 (1987).

4. S. Shigetomi, T. Ikari, Electrical and photovoltaic properties of $\mathrm{Cu}$-doped $p$-GaSe $/ n$-InSe heterojunction // Ibid. 88 (3), p. 1520-1524 (2000).

5. I.I. Grigorchak, V.V. Netjaga, S.V. Gavrilyuk, S.I. Drapak, Structure and physical properties of InSe and GaSe intercalated by molecular fluorine // Zhurnal Fizychnyh Doslidzhen' 6 (2), p. 193-196 (2002) (in Ukrainian).

6. Yu.I. Zhirko, I.P. Zharkov, Z.D. Kovalyuk, I.P. Shapovalova, Phonon spectra of doped and intercalated $\varepsilon$-GaSe crystals // Fizychnyj sbirnyk NTSh. 5 (4), p. 237-245 (2002) (in Ukrainian).

7. S.H. Anderson, D.D.L. Chung, Exfoliation of intercalated graphite // Carbon 22 (3), p. 253-263 (1984).

8. Z.D. Kovalyuk, T.P. Prokipchuk, A.I. Seredjuk, K.D. Tovstyuk, Investigation of impurity state in hydrogen intercalates of gallium selenium by NMR method // Fiz. Tverd. Tela 29 (7), p. 2191-2193 (1987).

9. Z.D. Kovalyuk, T.P. Prokipchuk, A.I. Seredjuk, K.D. Tovstyuk, S.Ja. Golub, V.I. Vitkovskaja, NMR in hydrogen subsystem of $\mathrm{H}_{x} \mathrm{GaSe}$ introduction junctions // Ibid. 30 (8), p. 2510-2511 (1988).

10. I.D. Kozmik, Z.D. Kovalyuk, I.I. Grigorchak, B.P. Bakchmatyuk, Preparation and properties oh hydrogen intercalated gallium and indium monoselenides // ISv. AN SSSR. Inorganic materials. 23 (5), p. $754-757$ (1987).

11. A. Polian, A. Kunc, A. Kuhn, Low-frequency lattice vibrations of $\partial$-GaSe compared to $\varepsilon$ - and $\gamma$ polytypes // Solid State Communs 19 (8), p. 10791082 (1976).

12. P.H. Ghosh, Vibrational spectra of layer crystals // Appl. Spectr. Rev. 19 (2), p. 259-323 (1983).

13. A. Kuhn, A. Chevy, R. Chevalier, Crystal structure and interatomic distance in GaSe // Phys. status solidi(b). 31 (2), p. 469-473 (1975). 
14. A. Goni, A. Cantarero, U. Schwarz, K. Syassen, A. Chevy, Low-temperature exciton absorption in InSe under pressure // Phys. Rev.B 45 (8), p. 4221-422 (1992).

15. D. Olguin, A. Cantarero, C. Ulrich, K. Suassen, Effect of pressure on structural properties and energy band gaps of $\gamma$-InSe // Phys. status solidi (b) 235 (2), p. 456-463 (2003).

16. Yu.I. Zhirko, Investigation of the light absorption mechanisms near exciton resonance in layered crystals. $N=1$ state exciton absorption in InSe // Ibid. 213 (1), p. 93-106 (1999).

17. Yu.I. Zhirko, Investigation of the light absorption mechanisms near exciton resonance in layered crystals. Part 2. $N=1$ state exciton absorption in GaSe // Ibid. 219 (1), p. 47-61 (2000).
18. R.J. Elliott, Intensity of optical absorption by excitons // Phys. Rev. 108, p. 1384-1389 (1957).

19. Y. Toyozawa, Theory of line-shapes of the exciton absorption bands // Progr. Theor. Phys. 20, p. 53-81 (1958).

20. C. Ulrich, D. Olguin, A. Cantarero, A.R. Goni, K. Syassen, A. Chevy, Effect of pressure on direct optical transitions of $\gamma$-InSe // Phys. status solidi (b) 221 (2), p. 777-787 (2000).

21. D. Errandonea, A. Segura, V. Munoz, A. Chevy, Pressure dependence of the low-frequency dielectric constant in III-VI semiconductors // Phys. status solidi (b) 211 (1), p. 201-206 (1999).

22. M.P. Malkov (ed.), Hand-book on physicalchemical fundamentals of cryogenics. Moscow, Energoatomizdat, 1985 (in Russian). 